\title{
Interaction of solitons in elongated BEC with time-dependent trap
}

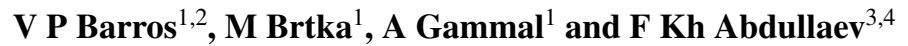 \\ ${ }^{1}$ Instituto de Física, Universidade de São Paulo, 05315-970, C.P. 66318 São Paulo, Brazil \\ 2 Departamento de Astronomia, Instituto de Astronomia, Geofísica e Ciências Atmosféricas, \\ Universidade de São Paulo, C.P. 3386, 01060-970 São Paulo, Brazil \\ 3 Dipartimento di Fisica 'E R Caianiello', Universitá di Salerno, I-84081 Baronissi (SA), Italy \\ ${ }^{4}$ Physical-Technical Institute of the Academy of Sciences, 700084, Tashkent-84, \\ G.Mavlyanov str., 2-b, Uzbekistan \\ E-mail: vpbarros@astro.iag.usp.br,mbrtka@if.usp.br,gammal@if.usp.br and \\ fatkhulla@yahoo.com
}

Received 13 June 2005, in final form 20 September 2005

Published 2 November 2005

Online at stacks.iop.org/JPhysB/38/4111

\begin{abstract}
The interaction of matter-wave solitons in elongated Bose-Einstein condensate with time-dependent parabolic trap is investigated using the perturbation theory based on the inverse scattering transform. Regimes of parametric and main resonances in solitons interactions are investigated for harmonic trap potentials. The predictions of the theory are confirmed by the numerical simulations of the quasi-one-dimensional Gross-Pitaevskii equation.
\end{abstract}

\section{Introduction}

Realization of bright matter waves in the laboratory has opened up an interest in solitary matter wave propagation [1,2]. In the experiment of [2] a bright matter-wave soliton train was created raising questions about the relative phase between solitons [3]. From soliton theory, it was first pointed out in $[4,5]$ that solitons in the nonlinear Schrödinger (NLS) equation with zero $(\pi)$ relative phase difference produce attractive (repulsive) interactions. Inverse scattering transform (IST) provided analytical solution to the soliton-soliton problem [6-9]. The same results can also be obtained from the variational approach [10]. In [7] it was shown that zero phase solitons develop an oscillating mode, whose frequency is well determined by soliton parameters.

Recently attention has been devoted to the theoretical studies of the soliton-soliton interactions in the trap potential. The energy exchange between solitons in the trap and the chaotic behaviour in oscillations have been investigated in [11, 12]. The interactions of two and $\mathrm{N}$-solitons in the harmonic trap were also analysed in $[13,14]$ from purposes of source of the generation and storage of matter-wave solitons train. A natural tool to investigate diverse 
dynamical behaviour of the system, in particular the presence of resonances, is to obtain the condensate response to temporal force as in $[15,16]$.

The main goal of this work is to investigate soliton-soliton interactions in the presence of a time-dependent harmonic trap. The time-dependent trap is used to analyse the collective oscillations of condensate [17]. Here we show that such modulations can help to obtain information about the matter-wave solitons interaction and give the possibilities of controlling the interactions. Note that the single bright soliton dynamics under a time-dependent trap were considered in [18-20]. The Schrödinger equation is integrable either with harmonic potential or with cubic nonlinearity. However, combination of both terms produce nonintegrable perturbed nonlinear Schrödinger model. We thus study the model by the perturbation theory based on the inverse scattering transform following $[4,21,22]$ and by full numerics solving directly the Gross-Pitaevskii equation.

The paper is organized as follows: in sections 2 and 3 we describe the model and analytical approaches based on the inverse scattering transform, in section 4 analytical and numerical results are presented and discussed, and finally in section 5 we summarize the main conclusions. In the appendix we derive the main equations using the variational approach.

\section{The model}

Matter waves in the Bose-Einstein condensate have been shown to be well described by the Gross-Pitaevskii equation which in three space dimensions has the form

$$
\mathrm{i} \hbar \Psi_{t}=-\frac{\hbar^{2}}{2 m} \nabla^{2} \Psi+\frac{m}{2}\left(\omega_{\perp}^{2} r_{\perp}^{2}+\omega_{x}^{2} x^{2}\right) \Psi+\frac{4 \pi \hbar^{2} a_{s}}{m}|\Psi|^{2} \Psi
$$

where $m$ is the atomic mass, $a_{s}$ is the interatomic scattering length, $a_{s}>0$ corresponds to the repulsive condensate and $a_{s}<0$ to the attractive one, $\omega_{\perp}$ is the radial frequency and $\omega_{x}$ is the axial frequency. The order parameter $\Psi$ is normalized to $N$, where $N$ is the number of the condensed atoms in the trap.

For an elongated trap with tight confinement in the transverse direction $\omega_{\perp} \gg \omega_{x}$ the quasi-one-dimensional(1D) Gross-Pitaevskii equation can be derived as [23]

$$
\mathrm{i} \hbar \psi_{t}+\frac{\hbar^{2}}{2 m} \psi_{x x}-g_{1 D}|\psi|^{2} \psi-\frac{m}{2} \omega_{x}^{2} x^{2} \psi=0
$$

where $g_{1 \mathrm{D}}=2 \hbar a_{s} \omega_{\perp}, a_{\perp}=\sqrt{\hbar /\left(m \omega_{\perp}\right)}$ and $\int|\psi|^{2} \mathrm{~d} x=N$. Below we will consider the case of attractive condensate $\left(a_{s}<0\right)$. In this case, the condition of validity of the quasi-1D approximation is

$$
N \frac{\left|a_{s}\right|}{a_{\perp}} \ll 1 .
$$

Typical experimental data are $\omega_{\perp}=2 \pi \times 640 \mathrm{~Hz}, \omega_{x}=2 \pi \times 3.2 \mathrm{~Hz}$, thus $\omega_{x} / \omega_{\perp}=a_{\perp}^{2} / a_{x}^{2} \sim 0.005$. The scattering length is $a_{s}=-0.16 \mathrm{~nm}$ [2]. The critical point when the collapse occurs is determined by the parameter $\kappa_{c}=N\left|a_{s}\right| / a_{\perp}=0.676$ $[23,24]$ (connected with the soliton amplitude $v$; see below).

To simplify the analysis, we introduce dimensionless variables $\omega=\omega_{x} / \omega_{\perp}, t^{\prime}=$ $t \omega_{\perp}, x^{\prime}=x / a_{\perp}, u=\sqrt{2\left|a_{s}\right|} \psi$ and we rewrite the equation as

$$
\mathrm{i} u_{t}+\frac{1}{2} u_{x x}+|u|^{2} u-\frac{1}{2} \omega^{2}(t) x^{2} u=0,
$$

where the primes on $t^{\prime}$ and $x^{\prime}$ are omitted for the convenience of notation. 


\section{Equations for two-soliton interactions in time-dependent trap}

In this paper, we study the interaction of two solitons when the trap is varying in time. As far as we know, this problem has not been considered previously and is of general interest as much for Bose-Einstein condensates (BEC) as for nonlinear optics. For two well-separated solitons (i.e., for the relative distance much larger that the solitons widths $a_{i}, i=1,2$ ), the solution is given approximately by

$$
u(x, t)=u_{1}(x, t)+u_{2}(x, t),
$$

where the single soliton solution is

$$
u_{n}(x, t)=2 v_{n} \operatorname{sech}\left[2 v_{n}\left(x-\xi_{n}\right)\right] \mathrm{e}^{\mathrm{i} 2 \mu_{n}\left(x-\xi_{n}\right)+\mathrm{i} \delta_{n}}
$$

with $n=1,2$. Substituting equation (5) into equation (4) and taking into account weak overlapping condition $\left(v_{1} \xi_{1}-v_{2} \xi_{2} \gg 1\right)$, we find the system

$$
\begin{aligned}
& \mathrm{i} u_{1, t}+\frac{1}{2} u_{1, x x}+\left|u_{1}\right|^{2} u_{1}=\frac{1}{2} \omega^{2} x^{2} u_{1}-\left(u_{2}^{*} u_{1}^{2}+2\left|u_{1}\right|^{2} u_{2}\right), \\
& \mathrm{i} u_{2, t}+\frac{1}{2} u_{2, x x}+\left|u_{2}\right|^{2} u_{2}=\frac{1}{2} \omega^{2} x^{2} u_{2}-\left(u_{1}^{*} u_{2}^{2}+2\left|u_{2}\right|^{2} u_{1}\right) .
\end{aligned}
$$

This system can be obtained from the Lagrangian

$$
\begin{gathered}
\mathcal{L}=\sum_{n=1,2}\left[\frac{\mathrm{i}}{2}\left(u_{n, t} u_{n}^{*}-\text { c.c. }\right)-\frac{1}{2}\left|u_{n, x}\right|^{2}+\frac{1}{2}\left|u_{n}\right|^{4}-\frac{1}{2} \omega^{2} x^{2}\left|u_{n}\right|^{2}\right] \\
-\mathrm{i} \epsilon\left(u_{1}^{*} R_{21}\left[u_{1}\right]+u_{2}^{*} R_{12}\left[u_{2}\right]+\text { c.c. }\right)
\end{gathered}
$$

where

$$
\epsilon R_{m n}\left[u_{n}\right]=\mathrm{i}\left(u_{m}^{*} u_{n}^{2}+2 u_{m}\left|u_{n}\right|^{2}\right)
$$

with $m, n=1,2, m \neq n$ and we must neglect $u_{n}$ dependence in $R_{m n}$ when taking the variational derivative. Since the overlap between solitonic tails is small and the trap potential is weak $\left(\omega^{2} \ll 1\right)$, we can use the perturbation theory based on the inverse scattering transform theory $[4,21,22]$. The perturbed NLS equation is

$$
\mathrm{i} u_{t}+\frac{1}{2} u_{x x}+|u|^{2} u=\mathrm{i} \epsilon R(u) .
$$

The equations for the soliton parameters are

$\frac{\mathrm{d} v}{\mathrm{~d} t}=\frac{\epsilon}{2} \operatorname{Re} \int_{-\infty}^{\infty} \frac{R\left[u_{s}(z)\right]}{\cosh (z)} \mathrm{e}^{-\mathrm{i} \theta(z, t)} \mathrm{d} z$

$\frac{\mathrm{d} \mu}{\mathrm{d} t}=\frac{\epsilon}{2} \operatorname{Im} \int_{-\infty}^{\infty} \frac{\tanh (z)}{\cosh (z)} R\left[u_{s}(z)\right] \mathrm{e}^{-\mathrm{i} \theta(z, t)} \mathrm{d} z$,

$\frac{\mathrm{d} \xi}{\mathrm{d} t}=2 \mu+\frac{\epsilon}{4 v^{2}} \operatorname{Re} \int_{-\infty}^{\infty} \frac{z R\left[u_{s}(z)\right]}{\cosh (z)} \mathrm{e}^{-\mathrm{i} \theta(z, t)} \mathrm{d} z$,

$\frac{\mathrm{d} \delta}{\mathrm{d} t}=2 \mu \frac{\mathrm{d} \xi}{\mathrm{d} t}+2\left(v^{2}-\mu^{2}\right)+\frac{\epsilon}{2 v} \operatorname{Im} \int_{-\infty}^{\infty} \frac{(1-z \tanh (z))}{\cosh (z)} R\left[u_{s}(z)\right] \mathrm{e}^{-\mathrm{i} \theta(z, t)} \mathrm{d} z$,

where $z=2 v(x-\xi(t)), \theta=(\mu / v) z+\delta$. As the result we find the evolution equations for the soliton parameters:

$$
\begin{aligned}
& \frac{\mathrm{d} v_{n}}{\mathrm{~d} t}=(-1)^{n} 16 v^{3} \mathrm{e}^{-2 v r} \sin (\phi), \\
& \frac{\mathrm{d} \mu_{n}}{\mathrm{~d} t}=-\frac{1}{2} \omega^{2} \xi_{n}+(-1)^{n} 16 v^{3} \mathrm{e}^{-2 v r} \cos (\phi),
\end{aligned}
$$


$\frac{\mathrm{d} \xi_{n}}{\mathrm{~d} t}=2 \mu_{n}+4 v \mathrm{e}^{-2 v r} \sin (\phi)$

$\frac{\mathrm{d} \delta_{n}}{\mathrm{~d} t}=-\frac{1}{2} \omega^{2}\left(\xi_{n}^{2}-\frac{\pi^{2}}{48 v_{n}^{2}}\right)+2\left(v_{n}^{2}+\mu_{n}^{2}\right)+8 \mu \nu \mathrm{e}^{-2 v r} \sin (\phi)+24 v^{2} \mathrm{e}^{-2 v r} \cos (\phi)$,

where $\phi=2 \mu r+\varphi, v=\left(v_{1}+v_{2}\right) / 2, \mu=\left(\mu_{1}+\mu_{2}\right) / 2, r=\xi_{1}-\xi_{2}, \varphi=\delta_{2}-\delta_{1}$. Here $r$ is the distance between solitons and $\varphi$ is the relative phase of solitons. It was assumed that $r>0$ and $\left|v_{1}-v_{2}\right| \ll v,\left|\mu_{1}-\mu_{2}\right| \ll \mu,\left|v_{1}-v_{2}\right| r \ll 1$ and $v r \gg 1$. Note that the variational approach in the appendix is free of the restriction for weak frequencies. For large distances the interaction term tends to zero and we get simply the harmonic oscillator. The restriction on the relative distance occurs from the requirement that the contribution from the trap is one order or more than the contribution from the solitons interaction, i.e., $\omega^{2} r \geqslant 64 v^{3} \exp (-2 v r)$. The adiabatic approximation considered in this work is valid for the time $\sim 1 / \epsilon$. The obtainment of a more accurate estimate for the time the calculation of the radiation is required, but that represents a separate problem.

From this system we obtain the equation for the relative distance of solitons

$$
\frac{\mathrm{d}^{2} r}{\mathrm{~d} t^{2}}=-\omega^{2}(t) r-64 v^{3} \mathrm{e}^{-2 v|r|} \operatorname{sgn}(r) \cos (\phi),
$$

where $\operatorname{sgn}(r)=1$ for $r>0$ and $\operatorname{sgn}(r)=-1$ for $r<0$. In the dimensional units the equation has the form

$$
\frac{\mathrm{d}^{2} \bar{r}}{\mathrm{~d} \bar{t}^{2}}=-\omega_{x}^{2}(t) \bar{r}-64 a_{\perp} \omega_{\perp}^{2} \nu^{3} \mathrm{e}^{-2 v|\bar{r}| / a_{\perp}} \operatorname{sgn}(r) \cos (\phi),
$$

where $v=N\left|a_{s}\right| /\left(2 a_{\perp}\right)$. This is the equation of motion of unit mass particle in anharmonic effective potential

$$
U(r)=\frac{1}{2} \omega^{2}(t) r^{2}-32 v^{2} \mathrm{e}^{-2 v|r|} \cos (\phi) .
$$

This potential has a discontinuity at $r=0$. The quasi-particle theory of Karpman-Solov'ev is valid when $v r \gg 1$. The equation for the relative phase is

$$
\frac{\mathrm{d}^{2} \phi}{\mathrm{d} t^{2}}=128 v^{4} \mathrm{e}^{-2 v|r|} \sin (\phi)
$$

\section{Analysis of the different regimes}

1. For the phase difference $\phi=0$ the interaction between solitons is attractive and in the dependence on the initial separation we have two oscillating regimes: in the first regime the harmonic trap is dominating and the frequency of oscillations is just given by the trap frequency. In the second the periodic coalescence of solitons dominates due to the interaction potential. When the trap is negligible, the solitons oscillate with the period [7]

$$
T \approx \frac{\pi}{4 v^{2}} \mathrm{e}^{v r_{0}}
$$

where $r_{0} \equiv r(t=0) v r_{0} \gg 1$. In general, the period of oscillations can be estimated from the potential of equation (21) and is given by

$$
T \approx 4 \sqrt{\frac{1}{2}} \int_{0}^{r_{0}} \frac{\mathrm{d} r}{\sqrt{\frac{1}{2} \omega^{2}\left(r_{0}^{2}-r^{2}\right)+32 v^{2}\left(\mathrm{e}^{-2 v r}-\mathrm{e}^{-2 v r_{0}}\right)}} .
$$

Although equation (21) is valid only for $v r \gg 1$, for small values of $r$ the solitons move relatively fast when compared to moderate and large $r^{\prime} s$ and there is little contribution to the period. Thus the lower limit of the integrand of equation (24) can be taken as zero. 


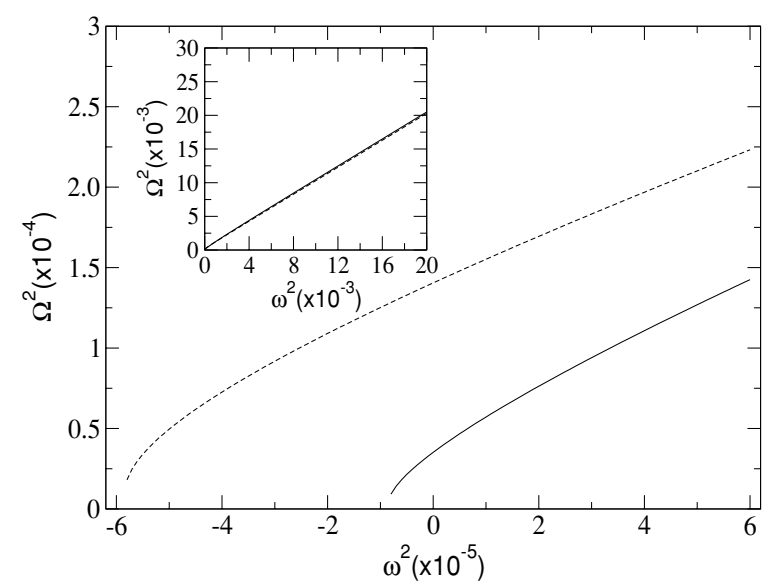

Figure 1. Soliton-soliton oscillation frequency perturbed by stationary harmonic trap. The phase difference is $\phi=0$ and relative distance is $r_{0}=20$. Dashed line corresponds to $v=0.10$ and solid line to $v=0.2$.

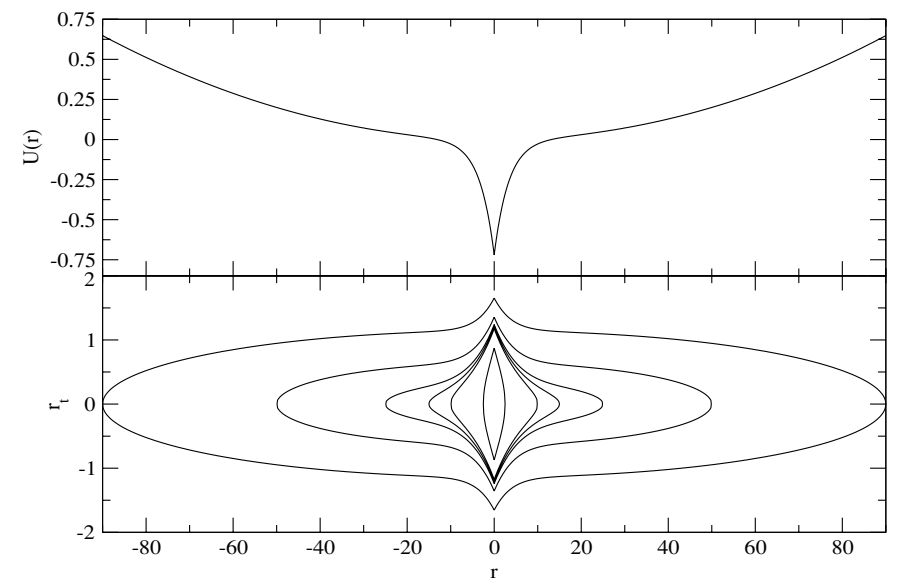

Figure 2. Potential $U(r)$ and phase space diagram for $\phi=0, v=0.15, \omega^{2}=1.6 \times 10^{-4}$. These plots are strictly valid only for $v r \gg 1$.

In figure 1 we present the numerical results of the oscillating frequency as a function of the trap frequency. For high trap frequencies, the trap almost entirely dominates the oscillating phenomena. For negligible trap $(\omega \sim 0)$ the numerics agree well with equation (23). Finally, for expulsive trap $\left(\omega^{2}<0\right)$ there is a critical trap frequency where the system stops oscillating. For $\omega^{2}$ below the squared critical frequency, the solitons are expelled. This demonstrates the richness of phenomena that can be controlled by external potential.

For the harmonic case $\left(\omega^{2}>0\right)$ we present the potential and phase space diagram in figure 2. Note that for large distances between solitons the potential $U(r)$ is almost harmonic.

In order to study a time-dependent trap, we considered the trap frequency varying in time in the form

$$
\omega^{2}=\omega_{0}^{2}\left[1+\eta \cos \left(\omega_{f} t\right)\right]
$$




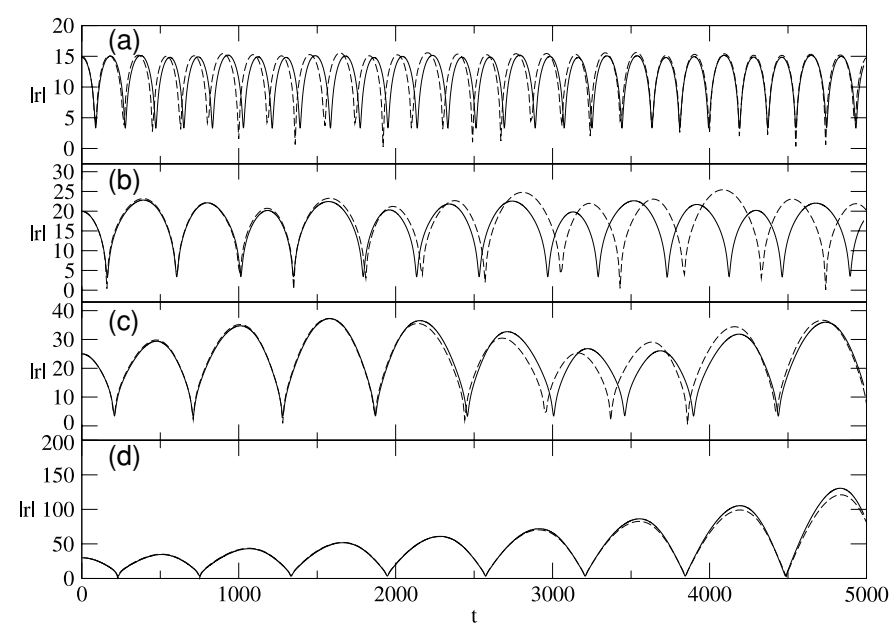

Figure 3. Oscillations of the relative distance $(r)$ for the zero relative phase case $\phi=0$ and different initial relative distances $r_{0}$. (a) $r_{0}=15$, (b) $r_{0}=20,(c) r_{0}=25,(d) r_{0}=30$; where $v=0.2, \omega_{0}=0.005, \omega_{f}=2 \omega_{0}, \eta=0.3$; PDE (solid lines) corresponds to equation (4) and ODE (dashed lines) to equation (26). In the PDE the distance of the solitons were obtained from the distance of centres of mass calculated for $(-\infty, 0]$ and for $[0, \infty)$.

and in this case equation (19) is modified for $r>0$ to

$$
\frac{\mathrm{d}^{2} r}{\mathrm{~d} t^{2}}+\omega_{0}^{2}\left[1+\eta \cos \left(\omega_{f} t\right)\right] r+64 v^{3} \mathrm{e}^{-2 v r} \cos (\phi)=0
$$

In figure 3 we present the results of the numerical simulations of the ODE and PDE for the case of $\omega_{f}=2 \omega_{0}$. As can be seen, for sufficiently large initial separation of the solitons $\left(r_{0}>r_{c} \sim 25\right)$, the parametric resonance in the oscillations is turned on, which leads to the growth of the relative distance. The inverse scattering transform results from ODE qualitatively agree with the full numerical simulations from PDE.

2. For the phase difference $\phi=\pi$ the interaction between solitons is repulsive and the effective potential, which is the sum of the trap and interaction potentials, has the form of the double well potential, i.e. equilibrium point at $r=0$ becomes unstable and two new equilibria points appear. The positions of minima are given by

$$
r_{1,2}= \pm r_{e}, r_{e}=\frac{64 v^{3} \mathrm{e}^{-2 v r_{e}}}{\omega^{2}}
$$

In figure 4 we show the potential $U(r)$ and the phase space diagram for $\phi=\pi$, using suitable parameters. The two lower points at the bottom of the double well correspond to the two fixed points. The fixed point in the oscillations of the relative distance $r$ corresponds to the stable bisoliton state, when the centres of both solitons are performing small oscillations near the stable points. For the phase difference $\phi=\pi$, we also consider the time-dependent trap frequency (25) and equation (26). In this case, the phase is oscillating with small amplitude near $\pi$ as

$$
\frac{\mathrm{d}^{2} \phi_{1}}{\mathrm{~d} t^{2}}+\Omega_{\phi}^{2} \phi_{1}=0
$$

where $\phi=\pi+\phi_{1}$, and with the frequency $\Omega_{\phi}=8 \sqrt{2} v^{2} \exp \left(-v r_{e}\right)=\sqrt{2 v r_{e}} \omega_{0}$, which is well separated from oscillations of the relative distance. Thus, in the linear approximation, 


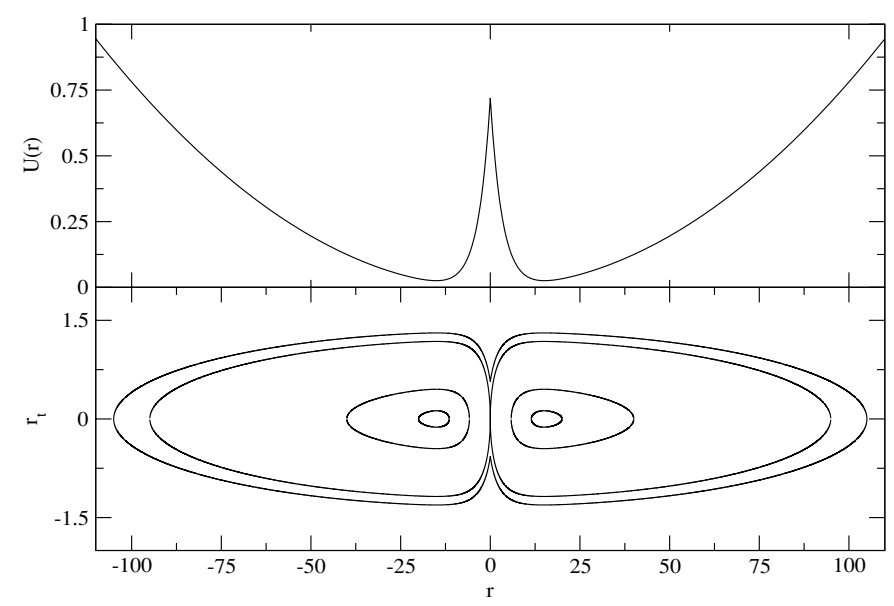

Figure 4. Potential $U(r)$ and phase space diagram for $\phi=\pi$ showing fixed points. $r_{e}=15$, $v=0.15, \omega=1.26 \times 10^{-2}$. These plots are strictly valid only for $v r \gg 1$.

the phase oscillations are decoupled from the oscillations of $r$. Then we can consider only the resonance in the relative distance oscillations.

For $\eta=0$ we have equilibrium points as given in (27). Substituting $y=r-r_{e}$ in (26), assuming $y$ small and using (27), we obtain

$$
\frac{\mathrm{d}^{2} y}{\mathrm{~d} t^{2}}+\omega_{0}^{2}\left[1+2 v r_{e}+\eta \cos \left(w_{f} t\right)\right] y=-\omega_{0}^{2} r_{e} \eta \cos \left(w_{f} t\right)
$$

One can rewrite this expression as

$$
\frac{\mathrm{d}^{2} y}{\mathrm{~d} t^{2}}+\bar{\omega}_{0}^{2}\left[1+\bar{\eta} \cos \left(\omega_{f} t\right)\right] y=F_{0} \cos \left(\omega_{f} t\right)
$$

where $\bar{\omega}_{0}^{2}=\omega_{0}^{2}\left[1+2 v r_{e}\right], \bar{\eta}=\eta /\left(1+2 v r_{e}\right)$ and $F_{0}=-\omega_{0}^{2} r_{e} \eta$. The solution for this equation is

$$
y(t)=y_{h}+y_{p}
$$

where $y_{h}$ and $y_{p}$ are respectively the homogeneous and particular solution to equation (30).

Using the standard approach [25] for the case of the parametric resonance where $\omega_{f}=2 \bar{\omega}_{0}+\varepsilon$, we find the following solution to the homogeneous equation:

$$
y_{h}(t)=\mathrm{e}^{\sigma t}\left[a_{0} \cos \left[\left(\bar{\omega}_{0}+\frac{\varepsilon}{2}\right) t\right]+b_{0} \sin \left[\left(\bar{\omega}_{0}+\frac{\varepsilon}{2}\right) t\right]\right],
$$

with $\sigma^{2}=\frac{1}{4}\left[\left(\frac{\bar{\eta} \bar{\omega}_{0}}{2}\right)^{2}-\varepsilon^{2}\right], a_{0}$ and $b_{0}$ being constants. In order to obtain the particular solution we employ the method of variation of parameters and find

$y_{p}(t)=\frac{F_{0}}{2 \bar{\omega}_{0}}\left[\frac{3 \bar{\omega}_{0} \cos \left(2 \bar{\omega}_{0} t\right)+\sigma \sin \left(2 \bar{\omega}_{0} t\right)}{\left(9 \bar{\omega}_{0}^{2}+\sigma^{2}\right)}-\frac{\bar{\omega}_{0} \cos \left(2 \bar{\omega}_{0} t\right)+\sigma \sin \left(2 \bar{\omega}_{0} t\right)}{\left(\bar{\omega}_{0}^{2}+\sigma^{2}\right)}\right]$.

Using the initial condition $y(t=0)=y_{0}, \mathrm{~d} y / \mathrm{d} t(t=0)=0$, the constants $a_{0}$ and $b_{0}$ are given by

$$
a_{0}=y_{0}-\frac{F_{0}}{2}\left[\frac{3}{\left(9 \bar{\omega}_{0}^{2}+\sigma^{2}\right)}-\frac{1}{\left(\bar{\omega}_{0}^{2}+\sigma^{2}\right)}\right]
$$




$$
b_{0}=-\frac{\sigma}{\bar{\omega}_{0}}\left\{y_{0}-\frac{F_{0}}{2}\left[\frac{1}{\left(9 \bar{\omega}_{0}^{2}+\sigma^{2}\right)}+\frac{1}{\left(\bar{\omega}_{0}^{2}+\sigma^{2}\right)}\right]\right\} .
$$

We performed numerical simulations on the diverse approaches including the PDE in the quasi-1D approximation. We used parameters $\omega_{0}=2.93 \times 10^{-3}$ and, $v=0.2, r(t=0)=20$ and $\eta=0.1$. The PDE numerical simulations were made using the Crank-Nicolson algorithm with initial profiles given by two solitons as given by equation (6).

We compared the numerical solutions of small amplitudes in the forced case (equation (30)) to the ones obtained analytically with the variation of parameters method equations (31)-(35). They showed remarkable agreement for small parameter $\bar{\eta}$. We also observed that the right-hand forcing term in equation (29) makes an enhancing of the amplitude of the resonance as compared to the standard Mathieu equation (figure 5(a)), and causes appearing of a second harmonic mode. This is in contrast with the standard Mathieu equation, where we have a single harmonic mode. In figure 5(b) we present the solution to equation (30) using the method of variation of parameters. In figure 5(c) we present the numerical solution of the soliton-soliton parametric resonance (equation (26)). A comparison of figures 5(b) and (c) shows remarkable agreement for small amplitudes. For growing amplitudes nonlinear terms of equation (26) have the effect of increasing the growing rate. Figure 5(d) shows the numerical solution of the PDE equation (2). The IST approach showed very good agreement with PDE results. In figure 5(e) we present the results from figures 5(a)-(d) superimposed for initial evolution. For longer times we observed that the shape of oscillations strongly deviates. Note the appearance of the second harmonic mode, a distinct effect from the standard Mathieu equation. In the main resonance case where $\omega_{f}=\bar{\omega}_{0}$, there is not closed approximation for the homogeneous solution of equation (30). To obtain an analytical approximation, we rewrite this equation as

$$
\hat{L} y(t)=S(y, t),
$$

where $\hat{L} \equiv \mathrm{d}^{2} / \mathrm{d} t^{2}+\bar{\omega}_{0}^{2}$ and $S \equiv\left[F_{0}-\bar{\omega}_{0}^{2} \bar{\eta} y(t)\right] \cos \left(\bar{\omega}_{0} t\right)$. In this form we can use Green's function standard iteration techniques [26]

$$
y_{(n)}(t)=y_{(0)}(t)+\int G(t-\tau) S\left(y_{(n-1)}(\tau), \tau\right) \mathrm{d} \tau .
$$

For the operator $\hat{L}$ the Green function is simply given by $G(t-\tau)=\sin \left[\bar{\omega}_{0}(t-\tau)\right] / \bar{\omega}_{0}$. Assuming $y(t=0)=y_{0}$ and $\mathrm{d} y / \mathrm{d} t(t=0)=0$ we have $y_{(0)}=y_{0} \cos \left(\bar{\omega}_{0} t\right)$ and up to second iteration we finally get

$$
\begin{aligned}
y_{(2)}(t)=y_{0}\left\{\cos \left(\bar{\omega}_{0} t\right)+\bar{\eta}\left[\frac{1}{3} \cos \left(\bar{\omega}_{0} t\right)+\frac{1}{6} \cos \left(2 \bar{\omega}_{0} t\right)-\frac{1}{2}\right]+\bar{\eta}^{2}\left[-\frac{1}{6}+\frac{87}{864} \cos \left(\bar{\omega}_{0} t\right)\right.\right. \\
\left.\left.+\frac{1}{18} \cos \left(2 \bar{\omega}_{0} t\right)+\frac{1}{96} \cos \left(3 \bar{\omega}_{0} t\right)+\frac{5}{24} \bar{\omega}_{0} t \sin \left(\bar{\omega}_{0} t\right)\right]\right\} \\
+F_{0}\left\{\frac{\bar{\eta}}{9 \bar{\omega}_{0}^{2}}\left[\cos \left(2 \bar{\omega}_{0} t\right)-\cos \left(\bar{\omega}_{0} t\right)\right]+\frac{\bar{\eta}}{12 \bar{\omega}_{0}} t \sin \left(2 \bar{\omega}_{0} t\right)+\frac{1}{2 \bar{\omega}_{0}} t \sin \left(\bar{\omega}_{0} t\right)\right\} .
\end{aligned}
$$

Our numerical studies showed remarkable agreement between the numerical solution of equation (30) and the Green's function approximation (38). In figure 6 we present the comparison of the numerical calculations of equation (26) and the Green's function approximation from equation (38). Clearly the approximation is good for short times but breaks down during the evolution. As the time evolves the amplitude increases and nonlinearity of equation (26) starts to play its role. This causes the effect of typical modulation of resonance, 

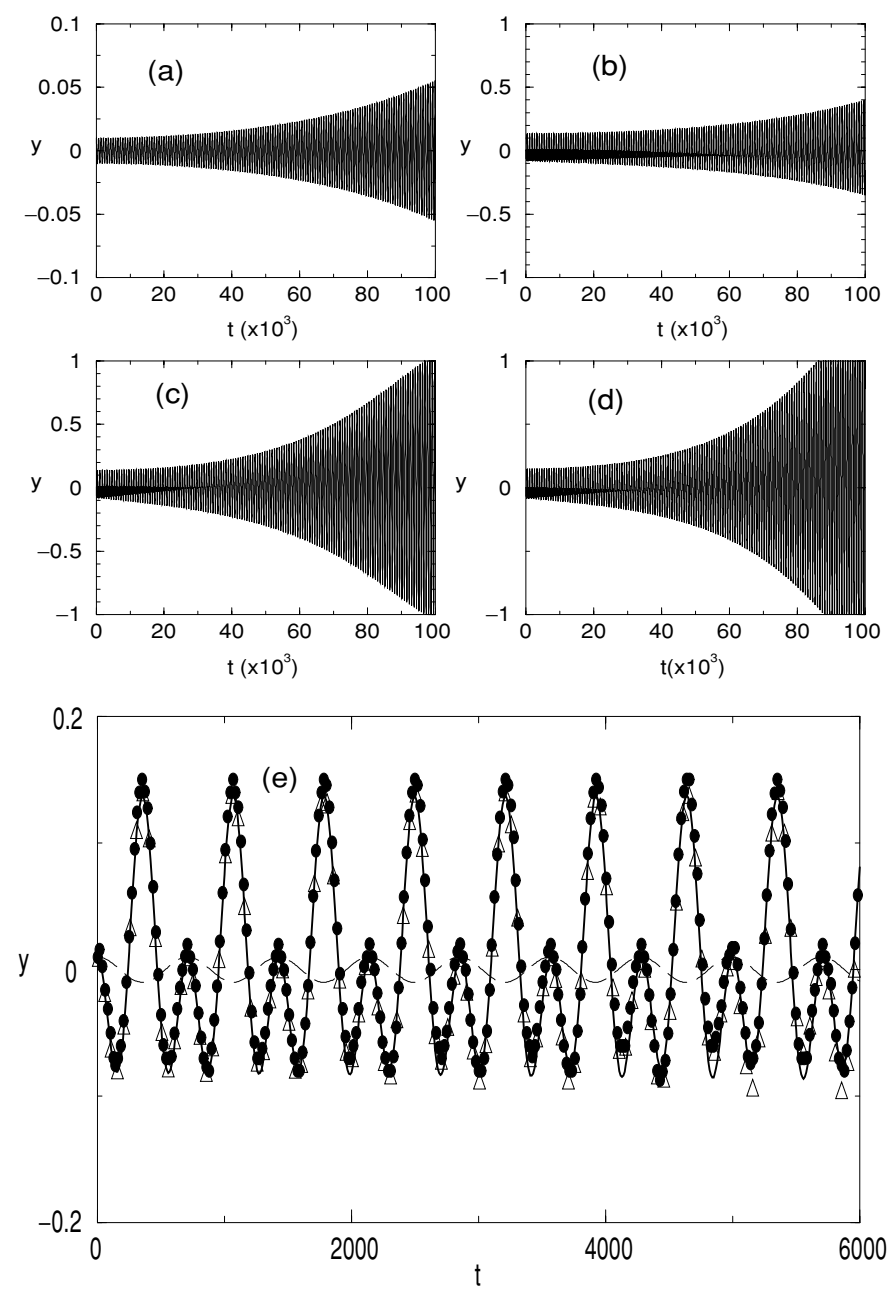

Figure 5. Parametric resonance in soliton-soliton interaction with $\omega_{0}=2.93 \times 10^{-3}, v=$ $0.2, r(0)=r_{e}=20, \eta=0.1$, case $\phi=\pi$. (a) Numerical simulation of the Mathieu equation given by equation (30) with $F_{0}=0$. (b) Analytical approximation obtained for small amplitude (equation (29)) using method of variation of parameters, equations (31)-(35). (c) Numerical simulation results of equation (26). (d) PDE results from simulation in the quasi-1D limit of equation (2). (e) results from figure 5(a) (dashed), (b) (solid), (c) (triangles), (d) (dots) superimposed for initial stages.

as we can observe. Let us estimate the value of these effects for the experimental case of [2]. For $v=0.2, r_{e}=20$ follows that $\bar{\omega}_{0}=\omega_{0} \sqrt{1+2 v r_{e}}=3 \omega_{0}$, so the parametric resonance occurs approximately at $\omega_{x}=2 \pi \times 19.2 \mathrm{~Hz}$ and the main resonance at $2 \pi \times 9.6 \mathrm{~Hz}$.

The resonant oscillations can lead to splitting of the bisolitonic state to separated solitons. Indeed, if the amplitude of oscillations of equation (34) becomes of order of the width of the potential $r_{m}$ at the maximum $U_{m}$, the bisoliton splits into two noninteracting solitons. The length $r_{m}$ can be estimated from $E_{\text {tot }}=U_{m}$, i.e.

$$
\frac{1}{2} \omega_{0}^{2} r_{m}^{2}=32 v^{2}\left(1-\mathrm{e}^{-2 v r_{m}}\right)
$$




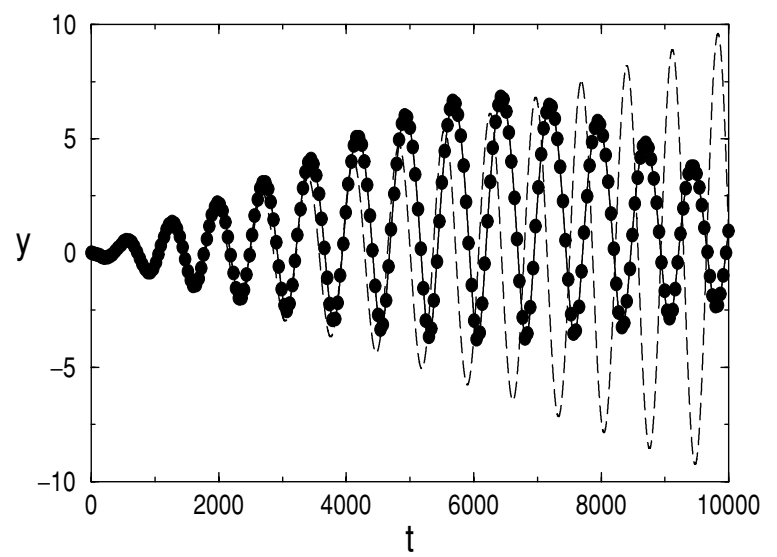

Figure 6. Main resonance in soliton-soliton interaction with $\omega_{0}=2.93 \times 10^{-3}, v=0.2, r_{0}=$ $20, \eta=0.1$. Dashed lines represent the analytical approximation obtained for small amplitude (equation (29)) using Green's function iterative method, equations (38). Solid line corresponds to numerical simulation results of equation (26). Filled dots is the solution of PDE in the quasi-1D regime, equation (2).

Thus $r_{m} \approx 8 v / \omega_{0}$. For the parameters of figure 4 it is $r_{m} \sim 95$. The maximum of amplitude at the parametric resonance at time $t=6000$ is $A_{m}<1$. The distortion of the bisoliton can happen if $A_{m}>r_{m}$. So this condition cannot be satisfied. The same conclusion is valid also for the main resonance case with $A_{m} \leqslant 10$ (see figure 5). Thus we can conclude that the bisoliton state in $\pi$-phase remains stable.

\section{Conclusion}

In conclusion, we have analysed the matter-wave soliton-soliton interactions in the elongated trap under the time-dependent modulations of the trap in the longitudinal direction. We developed the theory describing such interactions using the perturbation theory for solitons, based on the inverse scattering transform and the variational approach. We show that it is possible to obtain parametric and main resonances in the oscillations of the relative distance of solitons. The cases of phase differences zero and $\pi$ were considered. The analytical predictions are confirmed by the numerical simulations of the full Gross-Pitaevskii equation with the time-dependent trap. Future work can be the investigation of other regimes, in particular, the dynamical chaos caused by the interaction of solitons in time-dependent trap potential. The long-time evolution, when the interaction of solitons with emitted radiation becomes important, is another case which requires consideration beyond the quasi-particle picture used in this paper.

\section{Acknowledgments}

This work was supported by FAPESP (Brazil) and CNPq (Brazil). FKA is grateful to the University of Salerno for the research grant. 


\section{Appendix A}

Equations (15)-(18) can be alternatively obtained by a variational approach [10] to the averaged Lagrangian

$$
L=\int_{-\infty}^{+\infty} \mathcal{L}(x, t) \mathrm{d} x,
$$

where $\mathcal{L}$ is given by equation (8). Substituting (6) in (8) and then in (A.1) we obtain

$$
\begin{gathered}
L=\sum_{n=1,2} 8 v_{n} \mu_{n} \xi_{n, t}-4 v_{n} \delta_{n, t}-\frac{8 v_{n}^{3}}{3}-8 v_{n} \mu_{n}^{2}+\frac{16}{3} v_{n}^{3}-2 \omega^{2}\left[\frac{\pi^{2}}{48 v_{n}}+v_{n} \xi_{n}^{2}\right] \\
+96 v_{1}^{2} v_{2} \exp (-2 v r)+96 v_{1} v_{2}^{2} \exp (-2 v r) .
\end{gathered}
$$

Applying the Euler-Lagrange equations

$$
\frac{\partial L}{\partial q_{i}}-\frac{\mathrm{d}}{\mathrm{d} t} \frac{\partial L}{\partial q_{i, t}}=0
$$

where $q_{i}$ are the eight parameters $v_{1,2}, \mu_{1,2}, \delta_{1,2}$ and $\xi_{1,2}$, we obtain equation (15)-(18).

\section{References}

[1] Khaykovich L et al 2002 Science 2961290

[2] Strecker K E, Partridge G B, Truscott A G and Hulet R G 2002 Nature 417150

[3] Khawaja U Al, Stoof H T C, Hulet R G, Strecker K E and Partridge G B 2002 Phys. Rev. Lett. 89200404

[4] Karpman V I and Solov'ev V V 1981 Physica D 3142

[5] Gorshkov K A and Ostrovsky L A 1981 Physica D 3428

[6] Gordon J P 1983 Opt. Lett. 8596

[7] Desem C and Chu P 1992 Soliton-soliton interactions Optical Solitons: Theory and Experiment ed J R Taylor (Cambridge: Cambridge University Press) pp 107-51

[8] Hasegawa A and Kodama Y 1995 Solitons in Optical Communications (Oxford: Clarendon)

[9] Abdullaev F Kh, Darmanyan S A and Khabibullaev P K 1993 Optical Solitons (Heidelberg: Springer)

[10] Anderson D and Lisak M 1986 Opt. Lett. 11174

[11] Elyutin P V, Buryak A V, Gubernov V V, Sammut R A and Towers I N 2001 Phys. Rev. E 64016607

[12] Dmitriev S V, Semagin D A, Sukhorukov A A and Shigenari T 2002 Phys. Rev. E 66046609

[13] Carr L D and Brand J 2004 Phys. Rev. A 70033607

[14] Gerdjikov V S, Baizakov B B and Salerno M 2005 Theor. Math. Phys. 1441138

[15] Castin Y and Dum R 1996 Phys. Rev. Lett. 775315

[16] Ripoll J J G and Pérez-Garcia V M 1999 Phys. Rev. A 592220

[17] Jin D S, Esher J R, Matthews M R, Wieman C E and Cornell E A 1996 Phys. Rev. Lett. 77420

[18] Abdullaev F Kh and Galimzyanov R M 2003 J. Phys. B: At. Mol. Opt. Phys. 361099

[19] Abdullaev F Kh and Garnier J 2004 Phys. Rev. A 70053604

[20] Baizakov B, Filatella G, Malomed B A and Salerno M 2005 Phys. Rev. E 71036619

[21] Kaup D J 1976 SIAM J. Appl. Math. 31121

[22] Karpman V I and Maslov E M 1977 Sov. Phys. JETP 46281 Karpman V I and Maslov E M 1977 Zh. Eksp. Teor. Fiz. 73537 (in Russian)

[23] Pérez-Garcia V M, Michinel H and Herrero H 1998 Phys. Rev. A 573837

[24] Gammal A, Tomio L and Frederico T 2002 Phys. Rev. A 66043619

[25] Landau L D and Lifshitz E M 1975 Mechanics (Oxford: Pergamon)

[26] Byron F W and Fuller R W 1970 Mathematics of Classical and Quantum Physics vol 1 and 2 (Reading, MA: Addison-Wesley) 